\title{
Toward modeling turbulent suspension of sand in the nearshore
}

\author{
Tian-Jian Hsu \\ Applied Ocean Physics and Engineering, Woods Hole Oceanographic Institution, Woods Hole, Massachusetts, USA
}

Philip L.-F. Liu

School of Civil and Environmental Engineering, Cornell University, Ithaca, New York, USA

Received 10 December 2003; revised 5 April 2004; accepted 13 April 2004; published 18 June 2004.

[1] We present two depth- and phase-resolving models, based on single- and two-phase approaches for suspended sediment transport under water waves. Both models are the extension of a wave hydrodynamic model Cornell Breaking Wave and Structure (COBRAS). In the two-phase approach, dilute two-phase mass and momentum equations are calculated along with a fluid turbulence closure based on balance equations for the fluid turbulence kinetic energy $k_{f}$ and its dissipation rate $\epsilon_{f}$. In the single-phase approach the fluid flow is described by the Reynolds-Averaged Navier-Stokes equations, while the sediment concentration is calculated by an advection-diffusion equation for the conservation of sediment mass. The fluid turbulence is calculated by $k_{f}-\epsilon_{f}$ equations that incorporate the essential influence of sediment, which can also be consistently deduced from the two-phase theory. By adopting a commonly used sediment flux boundary condition near the bed the proposed models are tested against laboratory measurements of suspended sediment under nonbreaking skewed water waves and shoaling broken waves. Although the models predict wave-averaged sediment concentrations reasonably well, the corresponding time histories of instantaneous sediment concentration are less accurate. We demonstrate that this is due to the uncertainties in the near-bed sediment boundary conditions. In addition, we show that under breaking waves the near-bed sediment pickup cannot be solely parameterized by the bottom friction, suggesting that other effects may also influence the near-bed sediment boundary conditions. INDEX TERMS: 4546 Oceanography: Physical: Nearshore processes; 4558 Oceanography: Physical: Sediment transport; 4568 Oceanography: Physical: Turbulence, diffusion, and mixing processes; KEYWORDS: turbulent suspension, suspended sediment, pick-up function

Citation: Hsu, T.-J., and P. L.-F. Liu (2004), Toward modeling turbulent suspension of sand in the nearshore, J. Geophys. Res., 109, C06018, doi:10.1029/2003JC002240.

\section{Introduction}

[2] Sediment transport is usually caused by bottom friction, induced by near-bed shear flow, through particle intergranular interactions and fluid turbulent suspension. In the surf zone, additional turbulent suspension may be introduced by breaking wave turbulence. Because of the highly unsteady and complex hydrodynamic forcings and our limited understanding of sediment responses to these irregular forcings, modeling sediment transport processes in the nearshore remains to significantly rely on empiricism.

[3] While sediment transport in a major portion of water column may be caused by the fluid turbulence induced by boundary layer shear or wave breaking, the particle intergranular interaction is the major suspension mechanism in the concentrated sediment region near the bed. Within the surf zone, it is not clear, however, which mechanism contributes more to the total sediment transport because of the lack of detailed experimental evidence.

Copyright 2004 by the American Geophysical Union. 0148-0227/04/2003JC002240\$09.00
[4] In recent years, there has been increasing numbers of studies focusing on sediment transport in the highly concentrated near-bed region dominated by particle intergranular interactions. These models are either based on the Bagnold's relation [e.g., Dong and Zhang, 1999, 2002], the kinetic theory of granular flow [Jenkins and Hanes, 1998; Hsu et al., 2004] or the discrete element method [e.g., Drake and Calantoni, 2001]. Although these models are usually formulated for a relatively simple flow condition, such as uniform oscillatory flows, their results can still provide useful insight of the response of sediment bed under realistic nonbreaking waves. These models could also be used to provide near-bed sediment boundary conditions for large-scale sediment transport models.

[5] Sediment transport in the surf zone is further complicated by various nearshore hydrodynamic processes such as wave shoaling and wave breaking. In recent years, several surf zone hydrodynamic models based on depth-integrated equations, such as the nonlinear shallow water equations [e.g., Kobayashi and Johnson, 2001] and the Boussinesqtype equations [e.g., Wei and Kirby, 1995; Rakha et al., 1997; Lynett et al., 2002; Karambas and Koutitas, 2002], 
have been developed. These models have demonstrated a certain degree of success in modeling surf zone hydrodynamics and beach profile evolutions. However, since the vertical flow structure in these depth-integrated models are not resolved, the breaker location, energy dissipation and undertow profiles have to be further parameterized. Hence we believe that to advance our understanding on the interactions among wave breaking, near-bed flow and corresponding sediment responses in the surf zone we must rely on the phase- and depth-resolving models.

[6] One of the phase- and depth-resolving surf zone hydrodynamics models is based on the Reynolds-Averaged Navier-Stokes (RANS) equations along with a plausible turbulence closure and a robust free surface tracking scheme, such as the volume of fluid (VOF) method. Lin and Liu [1998a, 1998b] developed a numerical model, Cornell Breaking Wave and Structure (COBRAS) and modeled the wave breaking processes. They achieved satisfactory comparisons with laboratory measurements for the breaker location, the detailed flow and turbulence fields under both spilling and plunging breakers [Ting and Kirby, 1995, 1996].

[7] Because of the limitation on computational resources at the present time, in the sediment transport models that are used to simulate the entire surf zone, the concentrated sediment transport region near the bed can not be resolved. Hence near-bed sediment boundary conditions such as the sediment pickup function or the reference concentration need to be prescribed [e.g., Deigaard et al., 1986; Pedersen et al., 1995; Li and Davies, 1996; Savioli and Justesen, 1996; Duy and Shibayama, 1997]. At present, comprehensive tests for the available near-bed sediment boundary conditions for phase- and depth-resolving models under waves are still scarce.

[8] In this paper, we extend the wave hydrodynamics model COBRAS [Lin and Liu, 1998a, 1998b] to study the transport of sand under waves above the concentrated nearbed region using two different approaches. In the first approach, we employ the two-phase flow formulation and calculate the dilute two-phase mass and momentum equations for fluid and sediment phase, respectively. A closure of fluid turbulence, based on the transport equations of fluid turbulence kinetic energy $k_{f}$ and its dissipation rate $\epsilon_{f}$, is adopted [Hsu et al., 2003]. In the second approach, we employ the single-phase formulation. The original RANS equations of COBRAS are retained for fluid flow, while an advection-diffusion equation is incorporated for sediment concentration [e.g., Fredsøe and Deigaard, 1992; Nielsen, 1992; Duy and Shibayama, 1997]. Although the second approach can be viewed as a single-phase approach in terms of the mass and momentum equations, in the balance equations for fluid turbulent kinetic energy and its dissipation rate, the effects of sediment particles on the fluid turbulence are modeled through the frictional drag and density stratification [e.g., Hagatun and Eidsvik, 1986; McLean, 1992; Li and Davies, 1996]. We shall demonstrate that these additional terms can also be consistently deduced from the two-phase flow theory.

[9] In this paper, we conduct critical evaluation of the predictive capability of the proposed dilute sediment suspension models in terms of both the wave-averaged as well as the instantaneous sediment concentration. We demonstrate that these new models improve upon the earlier cross-shore sediment transport models of Pedersen et al. [1995] and Duy and Shibayama [1997] in terms of providing a complete two-equation turbulence closure, a more robust free surface tracking scheme for breaking waves based on the volume of fluid (VOF) method, and a better description of fluid-sediment interactions in the two-phase approach. However, in terms of predicting instantaneous suspended sediment concentration, large discrepancies remain. We shall show that these discrepancies are mainly due to the lack of accurate near-bed sediment boundary conditions.

[10] The paper is organized in the following manner. The balance equations and closures of both models are first summarized. Appropriate near-bed boundary conditions under waves are then discussed. Although the primary goal of this paper is to develop cross-shore sediment suspension models, we first test the models with the sheet flow experiments in a U tube by Ribberink and Al-Salem [1995]. This avoids the need to predict the hydrodynamics and focus attention on evaluating the sediment transport component of the proposed models. We then test the proposed models by comparing with the laboratory measurements of DohmenJanssen and Hanes [2002] conducted in a large wave flume under nonbreaking cnoidal waves. Finally, sediment suspension measurements under shoaling broken waves of Sato et al. [1990] are simulated. Through a discussion on the sediment diffusivities implemented in both models we explain the fundamental differences between the two approaches, which emphasizes the approximations embedded in the single-phase approach. We conclude the paper by an evaluation of the two-phase and single-phase approaches in terms of their accuracy and efficiency for nearshore sediment transport prediction.

\section{Model Formulation}

\subsection{Two-Phase Approach}

[11] Adopting the two-phase equations for dilute flow of Hsu et al. [2003], the Favre-averaged fluid phase and sediment phase continuity equations are

$$
\begin{gathered}
\frac{\partial(1-\bar{c})}{\partial t}+\frac{\partial(1-\bar{c}) \tilde{u}_{i}^{f}}{\partial x_{i}}=0 \\
\frac{\partial \bar{c}}{\partial t}+\frac{\partial \bar{c} \tilde{u}_{i}^{s}}{\partial x_{i}}=0
\end{gathered}
$$

where $i=1,2$ for the present two-dimensional models. In this paper, we further denote $x_{1}$ and $x_{2}$ by $x$ and $z$, respectively. $\bar{c}$ is the ensemble-averaged sediment concentration, with the overbar represents the ensemble-average operator. $\tilde{u}_{i}^{f}, \tilde{u}_{i}^{s}$ are the Favre-averaged (concentrationweighted) fluid-phase and sediment-phase velocities, defined as

$$
\tilde{u}_{i}^{f}=\frac{\overline{(1-c) u_{i}^{f}}}{(1-\bar{c})} \text { and } \tilde{u}_{i}^{s}=\frac{\overline{c u_{i}^{s}}}{\bar{c}}
$$

Therefore, in Favre averaging the averaged velocities are defined by the ensemble-averaged momentum fluxes and the volume fraction of the corresponding phase. The momentum 
equations of the fluid phase and sediment phase are written as

$$
\begin{gathered}
\frac{\partial(1-\bar{c}) \tilde{u}_{i}^{f}}{\partial t}+\frac{\partial(1-\bar{c}) \tilde{u}_{i}^{f} \tilde{u}_{j}^{f}}{\partial x_{j}}=-\frac{(1-\bar{c})}{\rho^{f}} \frac{\partial \bar{P}^{f}}{\partial x_{i}}-\frac{1}{\rho^{f}} \frac{\partial \tau_{i j}^{f}}{\partial x_{j}}+(1-\bar{c}) g_{i} \\
-\frac{\beta}{\rho^{f}} \bar{c}\left(\tilde{u}_{i}^{f}-\tilde{u}_{i}^{s}\right)-\frac{\beta \bar{c} \overline{\rho^{f}} \overline{c^{f}}}{}, \\
\frac{\partial \bar{c} \tilde{u}_{i}^{s}}{\partial t}+\frac{\partial \bar{c} \tilde{u}_{i}^{s} \tilde{u}_{j}^{s}}{\partial x_{j}}=-\frac{\bar{c}}{\rho^{s}} \frac{\partial \bar{P}^{f}}{\partial x_{i}}+\bar{c} g_{i}+\frac{\beta}{\rho^{s}} \bar{c}\left(\tilde{u}_{i}^{f}-\tilde{u}_{i}^{s}\right)+\frac{\beta}{\rho^{s}} \overline{c^{\prime} \Delta u_{i}^{f}},
\end{gathered}
$$

in which $\rho^{f}$ and $\rho^{s}$ are the density of fluid and sediment, $\bar{P}^{f}$ is the fluid pressure, $\tau_{i j}^{f}$ is the fluid phase stresses, including the viscous stress and Reynolds stress, and $g_{i}$ is the gravitational acceleration with $g_{1}=0.0$ and $g_{2}=-9.8\left(\mathrm{~m} / \mathrm{s}^{2}\right)$. Note that the terms related to the particle stresses, including the intergranular stress and large-scale sediment stress, in the sediment momentum equation (5) are neglected in a dilute flow [Hsu et al., 2003]. The last two terms in equations (4) and (5) are the Favre-averaged drag force with $\beta$ defined as

$$
\beta=\frac{18 \mu_{f}}{d^{2}(1-\bar{c})^{n}},
$$

where $\mu_{f}$ is the dynamic viscosity of the fluid, $d$ is the diameter of sediment particles. In this paper, sand of diameter about $0.2 \mathrm{~mm}$ is considered and the particle Reynolds number is assumed to be small. Hence only Stokes drag is incorporated for simplicity. The coefficient $n$ is a function of particle Reynolds number, taken to be 2.8 according to the experiments of Richardson and Zaki [1954]. We note here that the results are insensitive to the choice of $n$ for the present dilute flow. The last term in equations (4) and (5) is usually called fluid turbulent suspension with $c^{\prime}$ and $\Delta u_{i}^{f}$ the fluctuation components of concentration and fluid velocities relative to their ensemble mean $\bar{c}$ and $\tilde{u}_{i}^{f}$, respectively. Since we will adopt a fluid turbulence closure based on fluid phase eddy viscosity, $v_{f t}$, we shall calculate fluid turbulent suspension via the gradient transport assumption:

$$
\overline{c^{\prime} \Delta u_{i}^{f}}=-\frac{\nu_{f i}}{\sigma_{c}} \frac{\partial \bar{c}}{\partial x_{i}},
$$

with $\sigma_{c}$ being the Schmidt number.

[12] Since the fluid Reynolds stress is modeled using the eddy viscosity hypothesis, along with the fluid viscous stress, the total fluid stress can be written as:

$$
\begin{aligned}
\tau_{i j}^{f}= & \rho^{f}\left(v_{f t}+v_{f}\right)\left(\frac{\partial \tilde{u}_{i}^{f}}{\partial x_{j}}+\frac{\partial \tilde{u}_{j}^{f}}{\partial x_{i}}\right)-\frac{2}{3} \rho^{f}\left(v_{f t}+v_{f}\right) \frac{\partial \tilde{u}_{k}^{f}}{\partial x_{k}} \delta_{i j} \\
& -\frac{2}{3} \rho^{f}(1-\bar{c}) k_{f} \delta_{i j} .
\end{aligned}
$$

The fluid turbulent eddy viscosity $v_{f t}$ is related to fluid turbulence kinetic energy $k_{f}$ and its dissipation rate $\epsilon_{f}$ by:

$$
v_{f t}=C_{\mu} \frac{k_{f}^{2}(1-\bar{c})}{\epsilon_{f}}
$$

with $C_{\mu}$ being a numerical coefficient.
[13] According to Hsu et al. [2003], the balance equation for fluid turbulence kinetic energy can be written as:

$$
\begin{aligned}
\frac{\partial(1-\bar{c}) k_{f}}{\partial t}+\frac{\partial(1-\bar{c}) k_{f} \tilde{u}_{j}^{f}}{\partial x_{j}}= & \frac{\tau_{i j}^{f t}}{\rho^{f}} \frac{\partial \tilde{u}_{i}^{f}}{\partial x_{j}}+\frac{\partial}{\partial x_{j}}\left[\left(v_{f}+\frac{v_{f t}}{\sigma_{k}}\right)\right. \\
& \left.\cdot \frac{\partial(1-\bar{c}) k_{f}}{\partial x_{j}}\right]-(1-\bar{c}) \epsilon_{f} \\
& +\frac{\beta}{\rho^{f}} \frac{v_{f t}}{\sigma_{c}} \frac{\partial \bar{c}}{\partial x_{i}}\left(\tilde{u}_{i}^{f}-\tilde{u}_{i}^{s}\right) \\
& -2 \frac{\beta}{\rho^{f}} \bar{c} k_{f}(1-\alpha),
\end{aligned}
$$

and $\epsilon_{f}$ is assumed to be governed by another balance equation similar to that of $k_{f}$ :

$$
\begin{aligned}
\frac{\partial(1-\bar{c}) \epsilon_{f}}{\partial t}+\frac{\partial(1-\bar{c}) \epsilon_{f} \tilde{u}_{j}^{f}}{\partial x_{j}}= & C_{\epsilon 1} \frac{\epsilon_{f}}{k_{f}} \frac{\frac{f}{i j}^{f t}}{\rho^{f}} \frac{\partial \tilde{u}_{i}^{f}}{\partial x_{j}}+\frac{\partial}{\partial x_{j}}\left[\left(v_{f}+\frac{v_{f t}}{\sigma_{\epsilon}}\right)\right. \\
& \left.\cdot \frac{\partial(1-\bar{c}) \epsilon_{f}}{\partial x_{j}}\right]-C_{\epsilon_{2}} \frac{\epsilon_{f}}{k_{f}}(1-\bar{c}) \epsilon_{f} \\
& +C_{\epsilon_{3}} \frac{\epsilon_{f}}{k_{f}}\left[\frac{\beta}{\rho^{f}} \frac{v_{f t}}{\sigma_{c}} \frac{\partial \bar{c}}{\partial x_{i}}\left(\tilde{u}_{i}^{f}-\tilde{u}_{i}^{s}\right)\right] \\
& -C_{\epsilon_{3}} \frac{\epsilon_{f}}{k_{f}}\left[2 \frac{\beta}{\rho^{f}} \bar{c} k_{f}(1-\alpha)\right]
\end{aligned}
$$

The last two terms in equations (10) and (11) are additional sink (or source) for fluid turbulence due to the presence of sediment. Specifically, the last term represents a sink of fluid turbulence because the instantaneous velocity fluctuations of particles do not completely follow those of the fluid. Hence it is characterized by the degree of correlation between fluid velocity fluctuations and sediment velocity fluctuations [e.g., Young and Leeming, 1997]

$$
\alpha=\frac{T_{L}}{T_{L}+T_{p}}
$$

where $T_{p}=\rho^{s} / \beta$ is the particle response time and $T_{L}=$ $0.165 k_{f} / \epsilon_{f}$ is the fluid turbulence timescale.

[14] The numerical coefficients in equations (7), (9), (10) and (11) need to be specified. Employing the dilute assumption, we expect the modification of those coefficients due to the presence of sediment is small [e.g., Squires and Eaton, 1994], and we shall use the same coefficients as those suggested in the standard $k$ - $\epsilon$ model:

$$
C_{\mu}=0.09, \quad C_{\epsilon 1}=1.44, C_{\epsilon 2}=1.92, \quad \sigma_{k}=1.0, \quad \sigma_{\epsilon}=1.3 .
$$

The additional coefficient $C_{\epsilon 3}$ in the $\epsilon_{f}$ equation is taken to be 1.2, suggested by research on sediment-laden jets [Elghobashi and Abou-Arab, 1983]. The specification of the Schmidt number $\sigma_{c}$ is crucial in sediment transport and shall be discussed in later sections.

\subsection{Single-Phase Approach}

[15] In a typical single-phase approach, the sediment is considered to be dilute and passive. Here, the fluid flow is governed by the Reynolds-Averaged Navier-Stokes equations for a clear fluid. For the sediment phase the ensemble-averaged sediment velocity is assumed to follow 
the fluid velocity, except in the vertical direction the fall velocity $W_{o}$ are considered because of the immersed weight of sediments. Hence only the sediment concentration needs to be calculated and is assumed to be governed by an advection-diffusion equation [e.g., Fredsøe and Deigaard, 1992; Nielsen, 1992; Duy and Shibayama, 1997]:

$$
\frac{\partial \bar{c}}{\partial t}+\frac{\partial \bar{c} \tilde{u}_{j}^{f}}{\partial x_{j}}=\frac{\partial}{\partial x_{j}}\left(\frac{\nu_{f t}}{\sigma_{c}} \frac{\partial \bar{c}}{\partial x_{j}}\right)+\frac{\partial \bar{c} W_{o}}{\partial x_{j}} \delta_{2 j}
$$

The fall velocity $W_{o}$ needs to be further specified. Hsu et al. [2003] demonstrated that assuming that the vertical velocity of fluid and sediment in a fully developed flow (i.e., $\partial / \partial x=$ 0 ) is negligible, which is completely satisfied if the flow is also in steady state, the dilute two-phase equations can be reduced to those similar to Rouse [1937]:

$$
\bar{c} \frac{\left(\rho^{f}-\rho^{s}\right) g_{2}}{\beta}+\frac{\nu_{f t}}{\sigma_{c}} \frac{\partial \bar{c}}{\partial z}=0
$$

By further comparing equation (15) with the Rouse's [1937] formula, the fall velocity has the explicit form:

$$
W_{o}=\frac{\left(\rho^{f}-\rho^{s}\right) g_{2}}{\beta}
$$

with $\beta$ being calculated by equation (6).

[16] In the single-phase approach, even though it is assumed that the sediments do not directly affect the mean flow velocities through the momentum equations, the flow turbulence is usually considered to be modified by the sediments. Therefore, in the present formulation for fluid turbulence we seek for a simplified form for the $k-\epsilon$ equations based on equations (10) and (11). Consistent with the mean flow formulation, the difference between the horizontal velocities of the fluid and sediment phase is zero, i.e., $\tilde{u}^{f}-\tilde{u}^{S}=0$. However, the difference between the vertical velocities of the fluid and sediment becomes the fall velocity: $\tilde{w}^{f}-\tilde{w}^{s}=W_{o}$. Hence the 4th term on the righthand side of equation (10) becomes

$$
\beta \frac{v_{f t}}{\sigma_{c}} \frac{\partial \bar{c}}{\partial x_{i}}\left(\tilde{u}_{i}^{f}-\tilde{u}_{i}^{s}\right) \doteq-\left(\rho^{s}-\rho^{f}\right) g_{2} \frac{v_{f t}}{\sigma_{c}} \frac{\partial \bar{c}}{\partial z} .
$$

This term can be viewed as the so-called buoyancy production due to density stratification [e.g., McLean, 1992].

[17] In summary, in the present single-phase formulation, we solve the fluid flow by the Reynolds-Averaged NavierStokes equations and calculate the sediment concentration by the advection-diffusion equation (14). The fluid turbulent eddy viscosity is calculated by $k_{f}-\epsilon_{f}$ in equations (10) and (11) but with the fourth term on the right-hand side of each equation being replaced by equation (17).

\subsection{Near-Bed Boundary Conditions}

[18] In the models that simulate the sediment suspension on the entire cross-shore scale, it is not feasible to resolve the highly concentrated sediment region near the bed, whose thickness is of the order of a few grain diameters. Hence reasonable near-bed boundary conditions, which are able to appropriately describe the corresponding sediment response under various wave forcing and are consistent with the present phase- and depth-resolving models, must be specified.

[19] Because of the dilute assumption, the location of the conceptual "bed" in the present models is the interface between the concentrated sediment region and the dilute region where the particle intergranular stress becomes negligible. Obviously, the precise description of such interface can not be obtained in the present formulation. Hence the lower boundary of the present models is specified at a certain distance $\zeta$ above the initially undisturbed bed level. Typical estimates for the sheet flow layer thickness $\delta_{s}$ [Wilson, 1987; Sumer et al., 1996] are usually parameterized by the Shields parameter

$$
\theta=\frac{u_{*}^{2}}{(s-1) g d}
$$

where $u_{*}$ is the bed friction velocity, $g=\left|g_{2}\right|$, the magnitude of gravitational acceleration, and $s$ the sediment specific gravity. A Shields parameter about 2.0 gives a $\delta_{s}$ no more than about 20 grain diameters above the stationary bed level. Hence $\zeta$ is expected to be no more than about 10 grain diameters. For sand of typical diameter $0.2 \mathrm{~mm}, \zeta$ is only about 2 millimeters, which is much smaller than the vertical length scale of the water column in the surf zone. Therefore we consider such uncertainties in $\zeta$ inevitable but can be estimated approximately. In this paper, $\zeta$ is set to be equal to the roughness $K_{s}$, which shall be described in details next.

\subsubsection{Fluid Velocity and Bed Shear Stress}

[20] According to the measured fluid velocity profile above a mobile sand bed under steady and uniform flow condition, Sumer et al. [1996] suggested that the fluid velocity profile follows the logarithmic law:

$$
\frac{\tilde{u}^{f}}{u_{*}}=\frac{1}{\kappa} \ln \left[\frac{30 z}{K_{s}}\right] \text {, }
$$

where $\tilde{u}^{f}$ denotes the fluid velocity parallel to the bed, $\kappa=$ 0.41 is the Karman constant, and $K_{S}$ is the roughness. In the numerical implementation, we determine the friction velocity $u_{*}$ by evaluating equation (19) at the first grid point above the bed. The friction velocity is then used to calculate the bed shear stress, which in turns serves as the boundary condition for the fluid velocity and one of the important parameters for sediment near-bed boundary conditions.

\subsubsection{Fluid Turbulence}

[21] In the literature, two types of near-bed boundary conditions for fluid turbulence kinetic energy $k_{f}$ have been suggested. For example, Savioli and Justesen [1996] specified the boundary condition for $k_{f}$ in terms of friction velocity:

$$
k_{f}=\frac{u_{*}^{2}}{\sqrt{C_{\mu}}} .
$$

On the other hand, the no-flux boundary condition was adopted by others [e.g., Hagatun and Eidsvik, 1986; Hsu et al., 2003]:

$$
\frac{\partial k_{f}}{\partial z}=0
$$


On the basis of the two-phase theory, $k_{f}$ is affected by the sediment and is not known a priori. Moreover, in the surf zone, the near-bed fluid turbulence results from not only the bottom boundary layer but also the wave breaking processes. Using equation (20) in a relative coarse grid system near the bed accounts only for the bottom boundary layer turbulence and neglects the breaking wave generated turbulence. Therefore, in this paper, we adopt equation (21) as the bed boundary condition for $k_{f}$.

[22] The bed boundary condition for the turbulent dissipation rate $\epsilon$ is calculated by [e.g., Savioli and Justesen, 1996]

$$
\epsilon_{f}=\frac{C_{\mu}^{3 / 4} k_{f}^{3 / 2}}{\kappa z}
$$

\subsubsection{Sediment Flux}

[23] Sediment transport is initiated from the concentrated region near the bed where the dominate suspension mechanisms are particle intergranular stresses resulted from collisions, viscous suspension and enduring contact [e.g., Hsu et al., 2004]. In the present dilute models, such concentrated sediment region is not modeled and the only suspension mechanism is due to fluid turbulence. Therefore information on sediment vertical flux or pickup, which characterizes the amount of sediment suspended into the dilute region, is required.

[24] In the two-phase model, the sediment vertical flux $\bar{c} \tilde{w}^{s}$ needs to be specified as the near-bed sediment boundary condition. The parameterized formula that directly describe the sediment vertical flux under unsteady forcing is not yet available. Hence we assume that the near-bed sediment vertical flux can be expressed as the sum of an upward flux due to various suspension mechanisms, called pickup $\phi_{p}(\theta)$, and a downward flux due to the immersed weight of the sediment [Hsu et al., 2003]:

$$
\bar{c} \tilde{w}^{s}=\phi_{p}(\theta)+\frac{\left(\rho^{f}-\rho^{s}\right)}{\beta} \bar{c} g
$$

[25] In this paper, we adopt the sediment pickup formula suggested by van Rijn [1984a]:

$$
\frac{\phi_{p}(\theta)}{\sqrt{(s-1) g d}}=3.3 \times 10^{-4}\left[\frac{\theta-\theta_{c}}{\theta_{c}}\right]^{3 / 2}\left[\frac{(s-1) g d^{3}}{v_{f}^{2}}\right], \quad \theta>\theta_{c}
$$

$\theta_{c}=0.05$ the critical Shields parameter.

[26] In the single-phase model, the sediment pickup function is interpreted directly as the turbulent upward flux near the bed [e.g., Nielsen, 1992; Duy and Shibayama, 1997]:

$$
-\frac{v_{f t}}{\sigma_{c}} \frac{\partial \bar{c}}{\partial z}=\phi_{p}(\theta)
$$

Obviously, the accuracy of the predicted suspended sediment transport depends on a reasonable estimate of the Shields parameter $\theta$ in equation (24). For sediment transport in a $U$ tube or under nonbreaking waves, the Shields parameter is often calculated in terms of the bed friction velocity (equation (18)). Hence the sediment pickup at certain distance above the stationary bed depends only on bottom friction, while the bed friction velocity is in turn determined through equation (19) by the magnitude of nearbed velocity and the roughness $K_{s}$.

[27] On the other hand, near-bed sediment pickup flux under breaking waves can be enhanced by breaking wave induced turbulence, which may not be fully parameterized solely by bottom friction. A nondimensional parameter based on breaking wave turbulence energy $k_{f b}$ is introduced to parameterize the effects of breaking wave turbulence [e.g., Kobayashi and Tega, 2002]:

$$
\frac{e_{k} k_{f b}}{(s-1) g d}
$$

with $e_{k}$ being a numerical coefficient, which determines the sediment suspension efficiency. Hence we introduce a generalized Shields parameter to estimate the sediment pickup in the surf zone by combining equations (18) and (26):

$$
\theta=\frac{u_{*}^{2}+e_{k} k_{f b}}{(s-1) g d} .
$$

In the numerical implementation, since the generation and transport of the breaking waves turbulence is calculated from the $k_{f}-\epsilon_{f}$ equations, we adopt the calculated $k_{f}$ value at the first grid point above the bed for $k_{f b}$ in equation (27). Thus the intensity of wave breaking-induced turbulence on the near-bed sediment pickup is adjusted dynamically. However, the numerical value of $e_{k}$ is calibrated empirically on the basis of comparisons with the measured data.

\subsection{Numerical Implementation}

[28] The proposed equations are incorporated into COBRAS and are solved by a finite difference method. In the two-phase model, the sediment-phase mass and momentum equations are solved at the beginning of a computational cycle using a predictor-corrector scheme. After obtaining the sediment concentration and sediment velocities, the two-step projection method, which is a slightly modified version of the original COBRAS, is adopted to solve the fluid pressure and fluid velocities. The $k_{f}-\epsilon_{f}$ equations are then solved and the volume of fluid is updated at the end of the computational cycle, which follows the procedure of the original COBRAS. The details of the numerical scheme for two-phase flow are documented by Hsu [2002].

[29] In the single-phase model, the advection-diffusion equation of sediment is solved at the beginning of each computational cycle. The original RANS solver of COBRAS is retained to solve the fluid flow. In both approaches, the combined upwind and central difference method is adopted to discretize the advection terms.

\section{Results}

\subsection{Sand Transport in a U Tube}

[30] The proposed models are first tested with laboratory experiments of Ribberink and Al-Salem [1995] for sheet flow transport in a $U$ tube. Because the hydrodynamic 
(a)

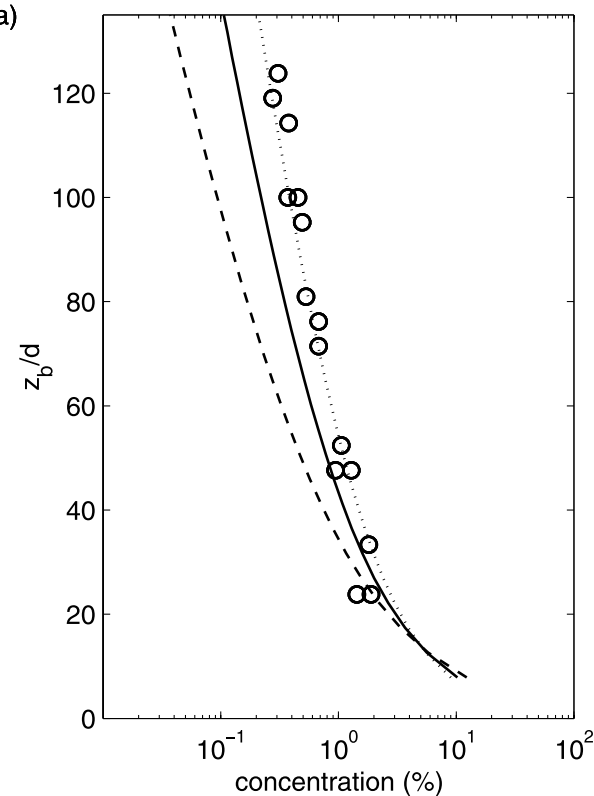

(b)

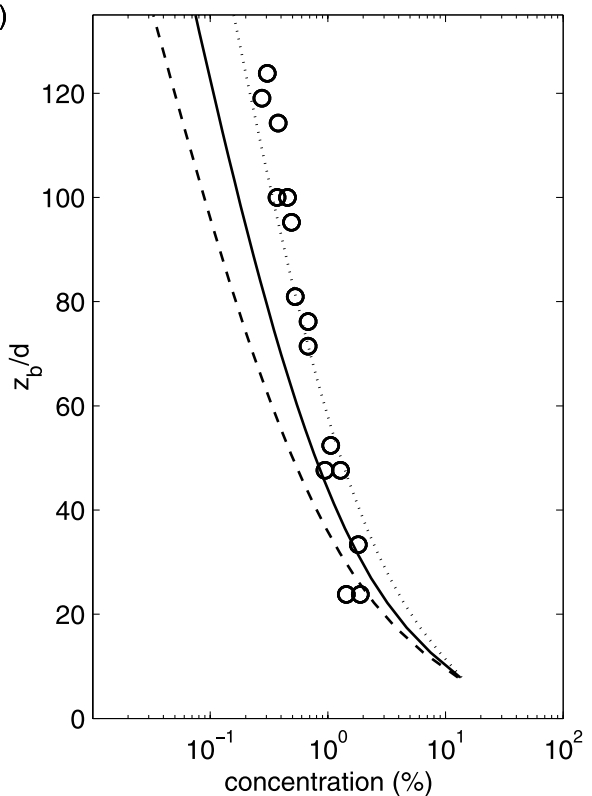

Figure 1. Comparison of wave-averaged sediment concentration with the measured data (symbols) of Ribberink and Al-Salem [1995] (case 3) using (a) the two-phase model with $\sigma_{c}=0.52$ (dotted curve), 0.7 (solid curve), and 1.0 (dashed curve), and (b) the single-phase model with $\sigma_{c}=0.35$ (dotted curve), 0.7 (solid curve), and 1.0 (dashed curve). The roughness is kept at $K_{s}=5.5 \mathrm{~d}$. For both the two-phase and single-phase models, $\sigma_{c}=0.7$ is the best fit value with suspended load data measured within $z_{b} / d=50$. The best fit value for $\sigma_{c}$ with all the suspended load data measured in the water column is 0.52 for twophase model and 0.35 for the single-phase model, respectively. Other U tube experiments [Horikawa et al., 1982; Staub et al., 1996] under sheet flow conditions are also tested, and their dependence on Schmidt number is similar.

forcing in a $U$ tube is a prescribed flow motion, by testing the model performance with the $U$ tube experiments, we avoid the uncertainties in predicting the correct hydrodynamic forcing and focus on examining the sediment transport component of the models and their near-bed boundary conditions.

[31] In the present models, because of the gradient transport assumption in fluid turbulent suspension (equation (7)) and the subgrid closure of the near-bed fluid velocity profile (equation (19)), the Schmidt number $\sigma_{c}$ and the roughness $K_{s}$ become two free parameters to be specified. Sumer et al. [1996] conducted a series of laboratory experiments of steady state, fully developed sheet flow sediment transport. For the cases of sand, the measured roughness $K_{s}$ is about 4 to 6 grain diameters and is relatively insensitive to the range of Shields parameter (from 0.83 to 2.24 ) tested. Hence we specify the roughness as $K_{s}=5.5 d$ to simulate the experiments of Ribberink and Al-Salem [1995]. We note that the roughness suggested by Sumer et al. [1996] has also been adopted by DohmenJanssen and Hanes [2002] in estimating the Shields parameters for their experiments.

[32] With the roughness fixed, we further calibrate the Schmidt number $\sigma_{c}$ for both two-phase and single-phase models using the measured wave-averaged sediment concentration of Ribberink and Al-Salem [1995] (case 3 of sinusoidal wave forcing of amplitude $U_{0}=1.7 \mathrm{~m} / \mathrm{s}$, oscillatory period $7.2 \mathrm{sec}$, and sand of diameter $d=$ $0.21 \mathrm{~mm}$, specific gravity $s=2.65$ ). Because a best fit (minimize the total error between the model results and measured data) of $\sigma_{c}$ with all the measured data points over the water column gives resulting calculated concentration profiles that overpredict the near-bed concentration by about a factor of two (Figure 1, dotted curves), we determine the optimal $\sigma_{c}$ by best fitting with the suspended data measured within fifty grain diameter above the initial bed level $\left(z_{b} / d<50\right)$ to emphasis the near-bed concentration, which facilitates our later attempts to evaluate the near-bed boundary condition presented next. A Schmidt number of $\sigma_{c}=0.7$ represents the best fit value for predicting the nearbed suspended concentration in both the two-phase (Figure 1a) and single-phase (Figure 1b) models.

[33] Although the present models, calibrated with values for roughness and the Schmidt number, estimate the waveaveraged sediment concentration profiles reasonably well (Figure 2a), their predictions of the time histories of instantaneous sediment concentration are less satisfactory (Figures $2 \mathrm{c}$ and 2d). In particular, the calculated time histories have more dramatic variations of magnitude over one wave cycle than those in the measured data. We believe that the discrepancies are mainly due to the adoption of steady state near-bed sediment boundary conditions to a unsteady flow problem. We remark that in van Rijn's [1984a] experiments for determining the pickup function, the sediment flux is measured under a steady clear fluid flow over a local supply of sand on an otherwise starved bed. Hence the sediment pickup is generated by a spatial inhomogeneity (not a temporal unsteadiness) with no upstream supply of sediments. When such a steady flow condition is further characterized by its corresponding bed 

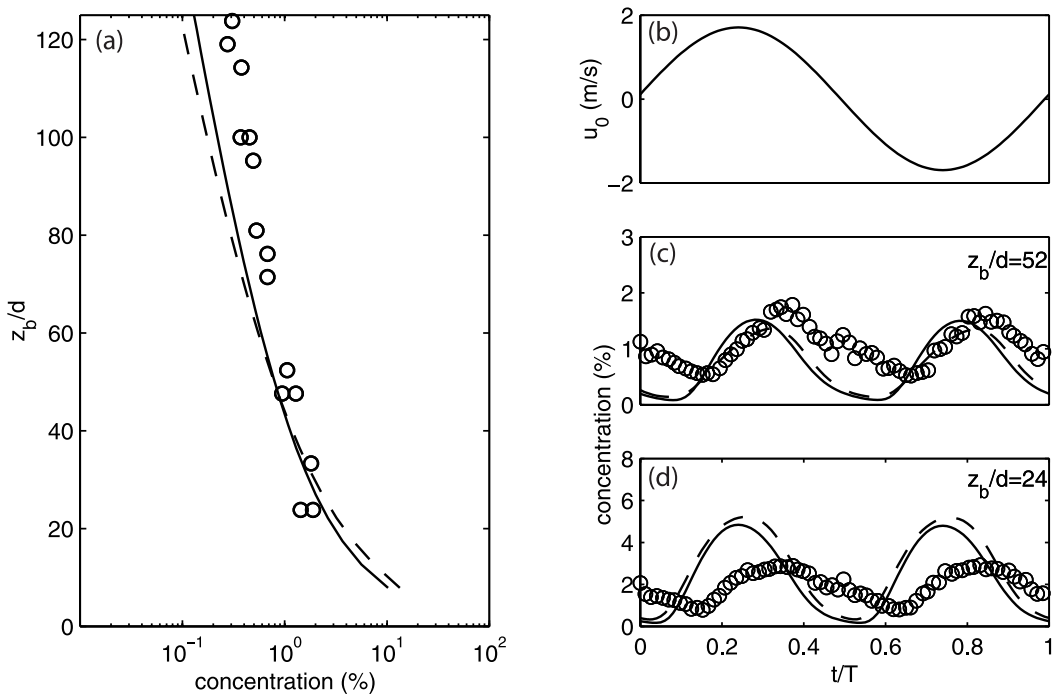

Figure 2. Comparison of sediment concentration between the two-phase model (solid curves), the single-phase model (dashed curves), and the measured data (symbols) of Ribberink and Al-Salem [1995] (case 3) with $K_{s}=5.5 d, \sigma_{c}=0.7$, using the pickup function of van Rijn [1984a] as near-bed sediment boundary condition: (a) wave-averaged sediment concentration, (b) time history of horizontal flow velocity at $25 \mathrm{~cm}$ above the bed, and (c) and (d) time histories of sediment concentration over one wave at two locations above the bed.

shear stress (i.e., the Shields parameter) and applied to unsteady flow in a quasi-steady sense, any memory effect of sediment transport, such as the hindered settling of sediments, is ignored. For instance, during the suspension phase $(t / T=0$ to 0.25 in Figures $2 \mathrm{~b}-2 \mathrm{~d})$, the pickup function may overpredict the sediment suspension flux without taking into account that the upstream supply of sediments may have already partially saturated the sediment boundary layer, resulting in an overprediction of instantaneous sediment concentration. On the contrary, during flow reversal where the flow velocity approaches zero $(t / T=0.4$ to 0.6 in Figures $2 b-2 d$ ), the pickup function would predict no sediment pickup without taking into account that it takes time for suspended sediments to settle back into bed [e.g., Fredsøe and Deigaard, 1992] and hence predicts a much lower concentration value during the flow reversal.

[34] In other words, we believe that the major reason for the poor performance of the sediment suspension models in predicting the time history of sediment concentration resulted from the inaccuracies of the near-bed boundary conditions and is not caused by the approximations in the governing equations and the corresponding closures. Such conjectures must be verified. Ribberink and Al-Salem [1995] also measure the time histories of sediment concentration in the concentrated region of sediment transport by the conductivity concentration meter (CCM). This allows us to use the measured sediment concentration time history $c_{b}(t)$ at the initial undisturbed bed level $\left(z_{b}=0\right)$ as the nearbed sediment boundary condition and recalculate case 3 using the two-phase model. The measured sediment concentration $c_{b}(t)$ at the initial bed level is shown in Figure 3e. Notice that over the entire wave cycle, the sediment concentration at this level is more or less a constant at about $20 \%$, suggesting that the sediment at this level does not response instantly to the free stream flow variations.
This observation is indeed consistent with the arguments made in the previous paragraph. In Figure 3a, using the twophase model with the same values for roughness and Schmidt number presented in Figure 2 and employing the new boundary condition $c_{b}(t)$, the predicted wave-averaged sediment concentration profile is almost identical to that by using van Rijn's pickup function as boundary condition. However, the calculated time histories of sediment concentration (Figures $3 \mathrm{~b}$ and $3 \mathrm{c}$ ) at two different elevations above the initial bed level agree very well with the measured data in both the magnitude and phase. Similar model performance is also achieved by using the single-phase model with the boundary condition $c_{b}(t)$ (not shown).

[35] Therefore we conclude that the approximations and the closures made in the governing equations are reasonable and the major inaccuracy in the present sediment suspension models comes from the near-bed sediment boundary conditions. Because of such limitations, the predictive capability of the present wave-resolving models are restricted to the wave-averaged sediment concentration. To further advance the performance of these wave-resolving sediment transport models, complete parameterizations for sediment pickup function or reference concentration should be conducted.

\subsection{Sand Transport Under Nonbreaking Waves}

[36] The proposed models are further tested with experiments under surface gravity waves. Dohmen-Janssen and Hanes [2002] generate cnoidal waves in a large wave flume with a uniform depth section of length $220 \mathrm{~m}$. The waves propagate (without breaking) over a horizontal sand bed $45 \mathrm{~m}$ long and $0.75 \mathrm{~m}$ thick positioned at about the central section of the flume. Testing the models with nonbreaking waves would add the effect of spatial inhomogeneity and vertical velocity, but, at the same time, would avoid the complications of the breaking wave turbulence on suspended 

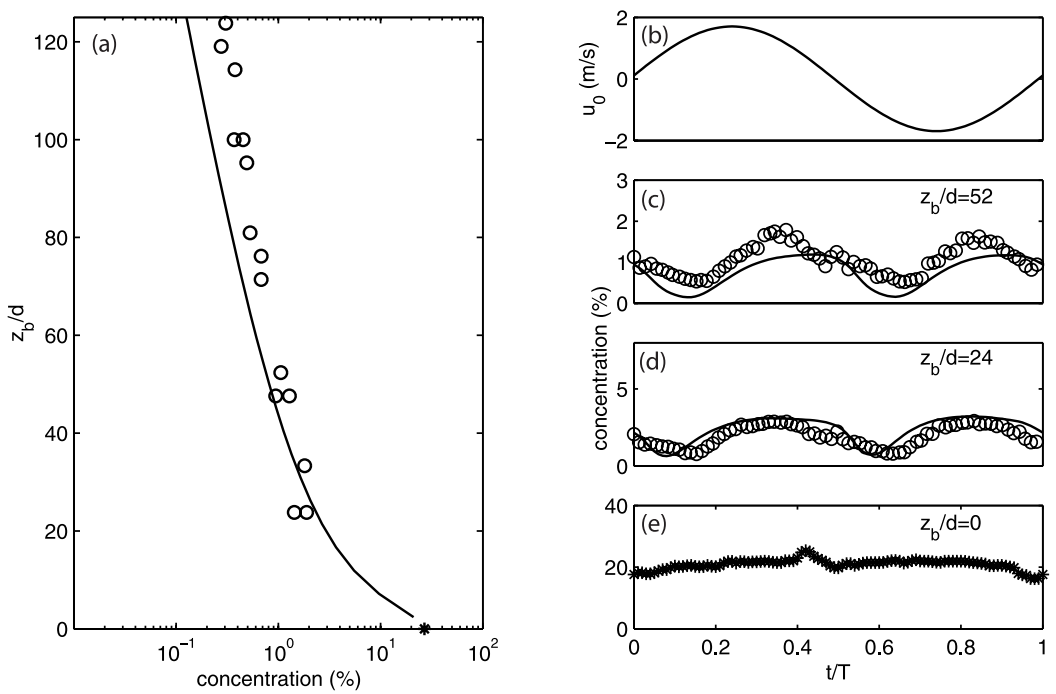

Figure 3. Comparison of the measured sediment concentration (symbols) of Ribberink and Al-Salem [1995] (case 3) with that of the two-phase model $\left(K_{s}=5.5 d, \sigma_{c}=0.7\right)$. The near-bed sediment boundary condition is prescribed on the basis of the measured sediment concentration time series $c_{b}(t)$ at initial undisturbed bed level (asterisks in Figures 3a and 3e). (a) Wave-averaged sediment concentration and (b) time history of horizontal flow velocity at $25 \mathrm{~cm}$ above the bed. (c) and (d) The predicted time histories of sediment concentration agree much better than that predicted by using the pickup function.

sediment transport. In the numerical simulation, the length of the computational domain is reduced to $165 \mathrm{~m}$ to save computational time. The entire computational domain is then discretized into $639 \times 144$ nonuniform grids with minimum grid sizes $\Delta x_{\min }=20 \mathrm{~cm}$ and $\Delta z_{\min }=0.2 \mathrm{~cm}$ in the central region of the test section. A roughness of $K_{s}=$ $5.5 d$ is adopted again.

[37] Using the two-phase model, the calculated waveaveraged sediment concentrations for case " $\mathrm{mh}$ " of sheet flow condition (incoming wave height $H=1.6 \mathrm{~m}$, wave period $6.5 \mathrm{sec}$, water depth at the wave maker $h=4.25 \mathrm{~m}$ and sand diameter $d=0.24 \mathrm{~mm}$, specific gravity $s=2.65$ ), are plotted against the measured data (Figure 4a). The measurement is conducted at the middle portion of the sand bed, which has a local water depth of 3.5 meter. Three different values of Schmidt number $\sigma_{c}=0.4,0.7,1.0$ are used and the numerical results suggest that Schmidt numbers of 0.7 are appropriate for the two-phase model. This is consistent with the results obtained previously for the $\mathrm{U}$ tube experiments. On the contrary, similar tests (Figure 4b) for the present single-phase model indicate that a Schmidt number value greater than unity needs to be used. We note here that Dohmen-Janssen and Hanes [2002] reported that the concentration measured in the very dilute region far
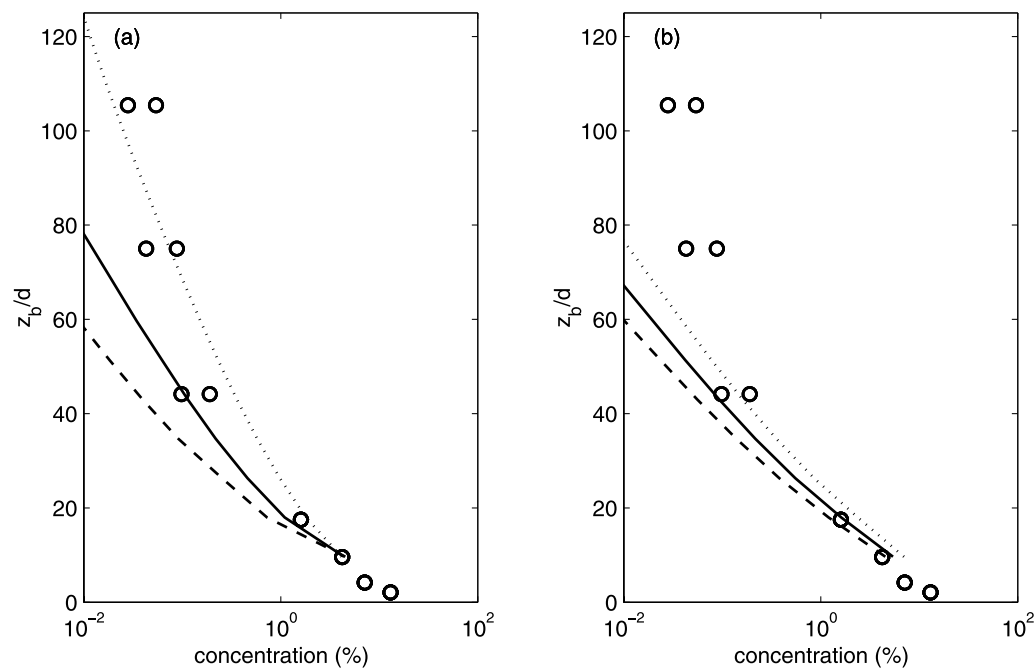

Figure 4. Comparison of wave-averaged sediment concentration with the measured data (symbols) of Dohmen-Janssen and Hanes [2002] (case "mh") using (a) the two-phase model with $\sigma_{c}=0.4$ (dotted curve), 0.7 (solid curve), and 1.0 (dashed curve) and (b) the single-phase model with $\sigma_{c}=1.0$ (dotted curve), 1.4 (solid curve), and 1.7 (dashed curve). The roughness is kept the same as $K_{s}=5.5 \mathrm{~d}$. 

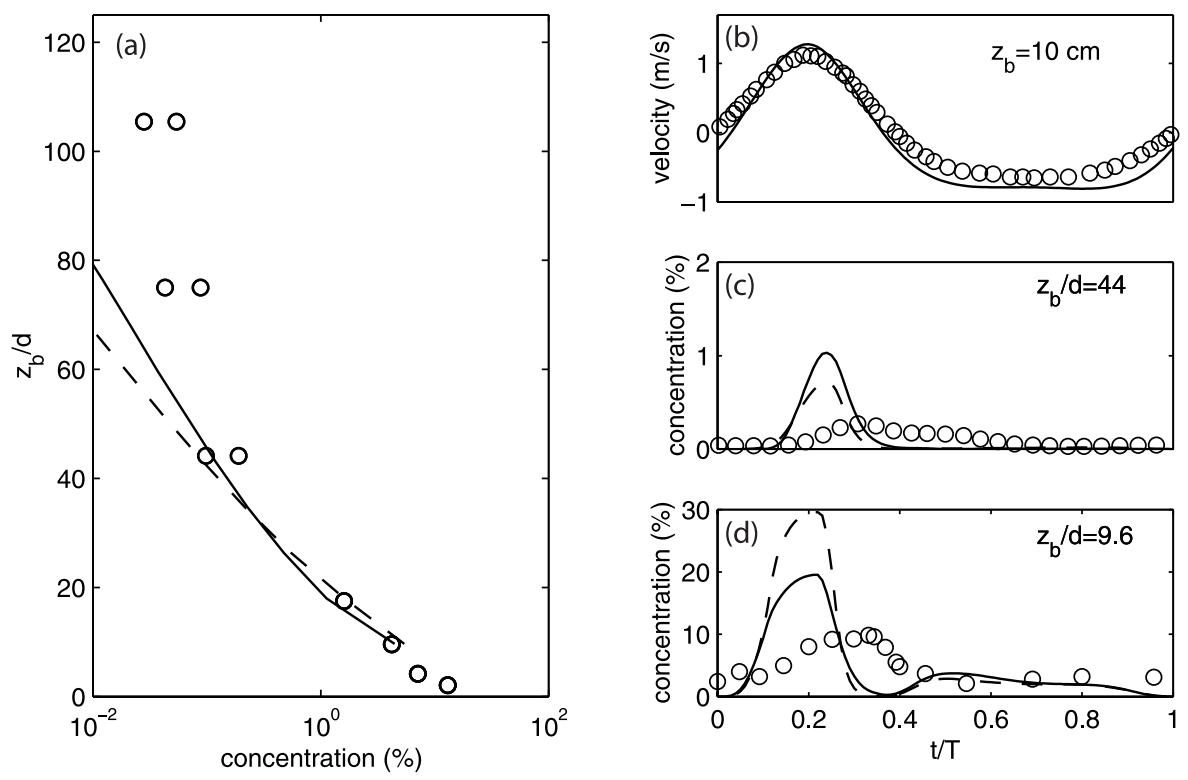

Figure 5. Comparison of measured sediment concentration of Dohmen-Janssen and Hanes [2002] (case "mh") (symbols) with the two-phase model (solid curves) $\left(K_{s}=5.5 d, \sigma_{c}=0.7\right)$ and single-phase model (dotted curves) $\left(K_{s}=5.5 d, \sigma_{c}=1.4\right)$ : (a) wave-averaged sediment concentration, (b) time history of horizontal flow velocity at $10 \mathrm{~cm}$ above the bed, and (c) and (d) time histories of sediment concentration over one wave at two locations above the bed.

from the bed may be influenced by the presence of very fine sediment and the background turbulence in the wave tank. Therefore measured concentration lower than about $0.05 \%$ may not be reliable.

[38] It seems that as far as the Schmidt number is concerned, the two-phase model is more robust for different type of flows, while in the single-phase model, significantly different values of Schmidt number are required. Further comparisons between Figures $4 \mathrm{a}$ and $4 \mathrm{~b}$ suggest another important difference of the Schmidt number effect on both models. In the two-phase model, the Schmidt number modifies the overall slope of the calculated sediment concentration profile while the magnitude of the concentration near the bed is relatively insensitive to the Schmidt number. On the other hand, the Schmidt number changes the magnitude of the entire concentration profile more uniformly in the singlephase approach.

[39] Again by examining the calculated time histories of sediment concentration using the pickup function as the boundary condition, we conclude that both the two-phase approach (solid curves) and the single-phase approach (dotted curves) predict poorly (Figures $5 \mathrm{c}$ and $5 \mathrm{~d}$ ), even though the predicted flow velocity under waves (Figure 5b) agree with experimental data reasonably well. The features of the computed instantaneous concentration profiles are similar to those in the $U$ tube tests presented in Figure 2. In the next section, we shall only focus on wave-averaged sediment concentration when further testing the models with sediment transport measurements under breaking waves.

\subsection{Sand Transport Under Shoaling Breaking Waves}

[40] The proposed models are further tested with laboratory measurements of Sato et al. [1990] on suspended sand concentration under breaking waves in the surf zone. the wave flume of Sato et al. [1990] has a total length of $18 \mathrm{~m}$ and a 1/20 impermeable beach is installed at the end of the wave flume. The length of the uniform water depth section is $8 \mathrm{~m}$. Near the wave breaking point, the solid sloping bottom is replaced by a sediment tray that has $1 \mathrm{~m}$ in length and $2 \mathrm{~cm}$ in depth, filled up with sand of specific gravity $s=$ 2.65 and diameter of either $d=0.15 \mathrm{~mm}$ or $0.18 \mathrm{~mm}$. In the numerical simulations, the entire computational domain is discretized into $998 \times 88$ nonuniform grids with minimum grid sizes $\Delta x_{\text {min }}=1 \mathrm{~cm}$ and $\Delta z_{\text {min }}=0.4 \mathrm{~cm}$ in the vicinity of the wave breaking point. The wave condition studied here has a wave height $H=9.9 \mathrm{~cm}$, and wave period $T=1.35 \mathrm{sec}$ and are generated in a uniform water depth of $h=39.5 \mathrm{~cm}$.

[41] The accuracy of COBRAS for surf zone hydrodynamics has been reported by Lin and Liu [1998a, 1998b, 2004], where detailed time histories of flow velocity, turbulence and undertow profiles at various locations within the surf zone are compared with laboratory measurements of Ting and Kirby [1995, 1996]. The computed mean free surface, mean crest and mean trough level (Figure 6a), and undertow profiles (Figure 6b) for Sato's experiment demonstrate reasonable predictive ability of the present model for surf zone hydrodynamics. According to Figure 6a, the predicted wave breaking point is at about $x=13.2 \mathrm{~m}$. To facilitate our illustration for the surf zone, we define another coordinate system $\chi$, where $\chi=0$ is the location of shoreline at the still water level. Thus a surf zone length $l_{b}$, defined as the distance between the shoreline and the wave breaking point, of $2.7 \mathrm{~m}$ is obtained.

[42] Using the two-phase approach (with $\sigma_{c}=0.7$, calibrated with nonbreaking wave case in the previous section), the predicted wave-averaged suspended sediment concentration profiles at three locations within the surf zone 

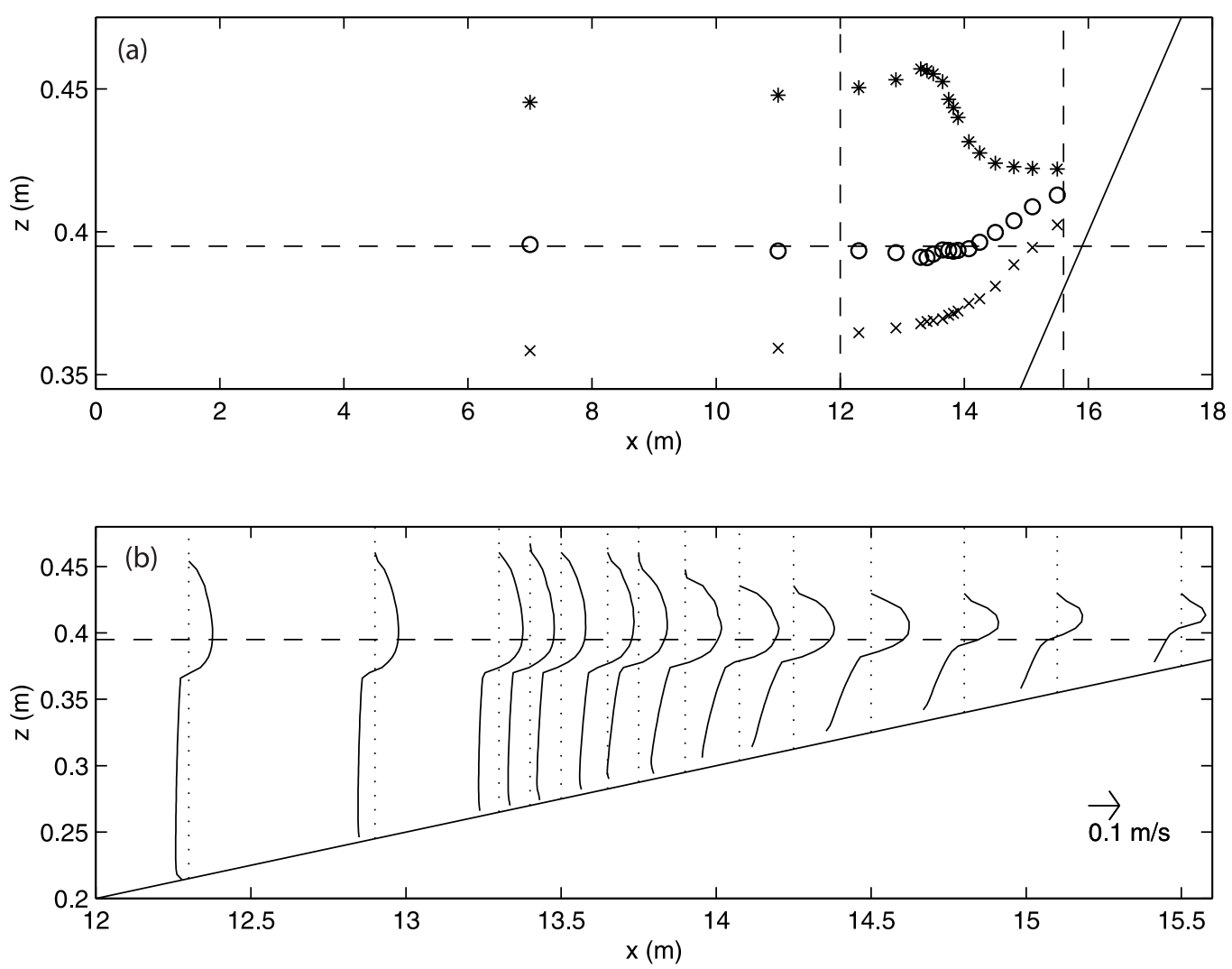

Figure 6. Calculated (a) mean water level $(O)$, crest level $(*)$, and trough level $(\times)$ and $(b)$ waveaveraged horizontal fluid velocity across the surf zone of Sato et al. [1990] with wave height $H=9.9 \mathrm{~cm}$, uniform water depth $h=39.5 \mathrm{~cm}$, and wave period $T=1.35 \mathrm{~s}$. The time averagings for the mean quantities are performed for the last four waves after quasi-steady state.

$\left(\chi / l_{b}=0.86,0.8,0.73\right)$ are compared with the measured data for the case of sand with diameter $d=0.15 \mathrm{~mm}$ (Figure 7). When equation (18) is used to calculate the Shields parameter for sediment pickup (dashed curve), which only accounts for the effect of bottom friction, the predicted sediment concentration is about one order of magnitude smaller than the measured data. On the other hand, when equation (27) with $e_{k}=0.05$ is used so that the effect of breaking wave turbulence is also taken into account for estimating the pickup function, the predicted wave-averaged sediment concentrations agree fairly well with the measured data (solid curve). In Figure 8, results computed from the single-phase approach (with $\sigma_{c}=1.4$ ) for the same case also leads to a similar conclusion that the incorporation of breaking wave turbulence (with $e_{k}=0.018$ ) into the calculation of the sediment pickup may be necessary. Hence, on the basis of the present model the sediment pickup under breaking waves is not only influenced by the bottom friction but also enhanced by the breaking wave turbulence.

[43] The case of larger grain size of $0.18 \mathrm{~mm}$ is further tested. Using the numerical coefficient $e_{k}$ determined from the previous case (i.e., $e_{k}=0.05$ and 0.018 for two-phase and single-phase approaches, respectively), the computed results (Figure 9) from the two-phase model (solid curve) and single-phase model (dashed curve) agree fairly well with the measured data. Although we have demonstrated that the value of $e_{k}$ in each model is insensitive to grain sizes, our numerical simulations only show qualitatively the importance of the breaking wave generated turbulence on near-bed sediment pickup because of the uncertainties in the near-bed boundary conditions as discussed before. More comprehensive measurements on the interactions between the breaking wave generated turbulence and near-bed sediment response are clearly needed.

\subsection{Discussion on Sediment Diffusivity}

[44] Within the context of the ensemble-averaged approach, we have adopted perhaps the simplest closure on fluid turbulent suspension based on the gradient transport assumption (equation (7)), where the sediment diffusivity is proportional to the eddy viscosity. Evidently, the gradient transport assumption has a great advantage of easy implementation as the turbulent eddy viscosity is already calculated in the turbulence model. Nevertheless, it requires the specification of the Schmidt number $\sigma_{c}$. In this paper, we treat the Schmidt number as a free parameter and calibrate it with the measured data and obtain different values for the two-phase and single-phase approach. Particularly, the calibrated $\sigma_{c}$ in the two-phase approach gives a value smaller than unity in both the $U$ tube flow and surface gravity waves, suggesting that the turbulent diffusion of sediment is more efficient than that of fluid momentum. On the other hand, the calibrated $\sigma_{c}$ value in the single-phase approach 

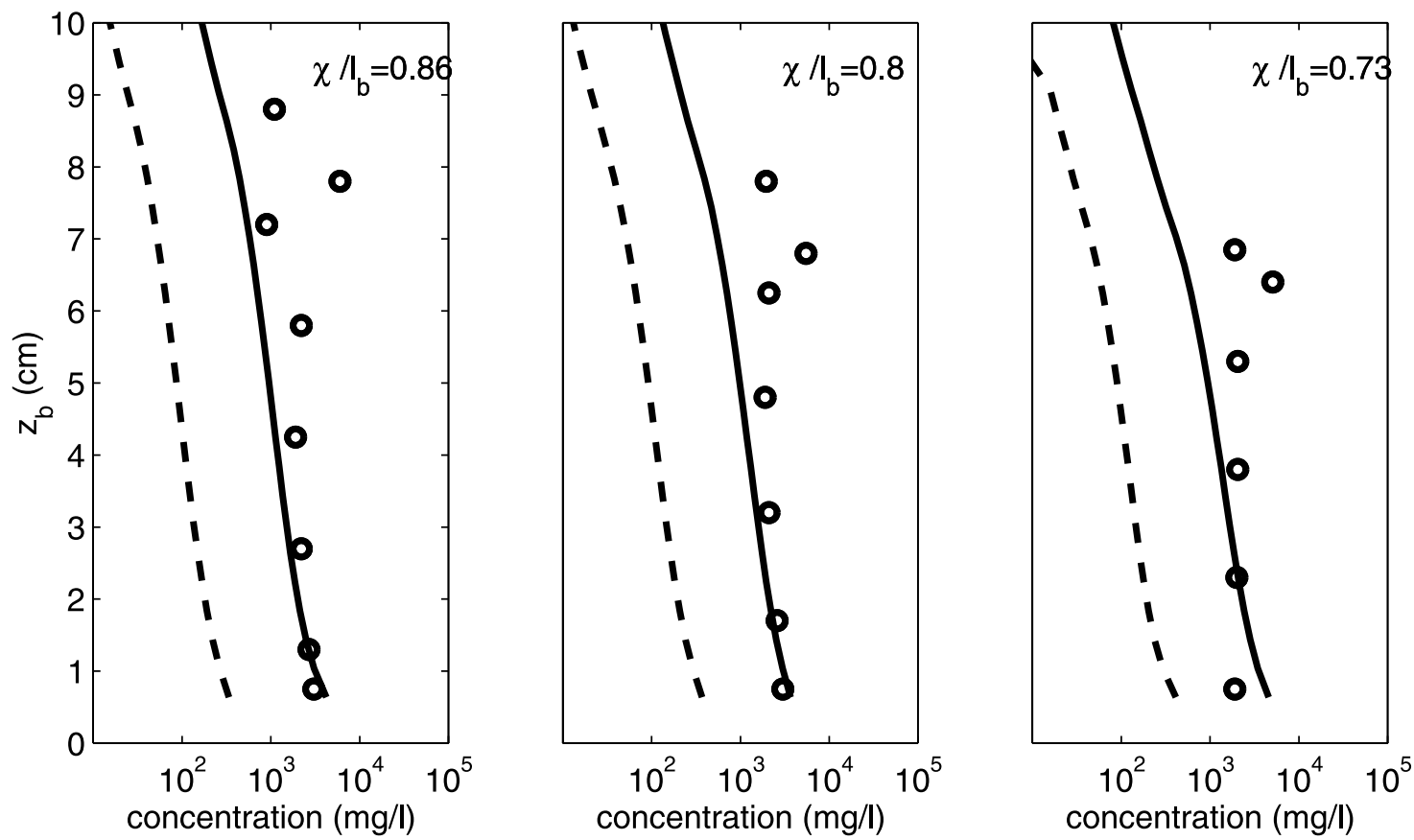

Figure 7. Comparisons of wave-averaged sediment concentration profiles using the two-phase model at three locations within the surf zone of Sato et al. [1990] with the same wave condition as in Figure 6 and sand of diameter $d=0.15 \mathrm{~mm}$. Open circles represent the measured data. Estimating the near-bed pickup using only the bottom friction underpredicts the suspended sediment concentration $\left(e_{k}=0.0\right.$, dashed curves). Further incorporating the effect of breaking wave turbulence ( $e_{k}=0.05$, solid curves) gives better agreements.
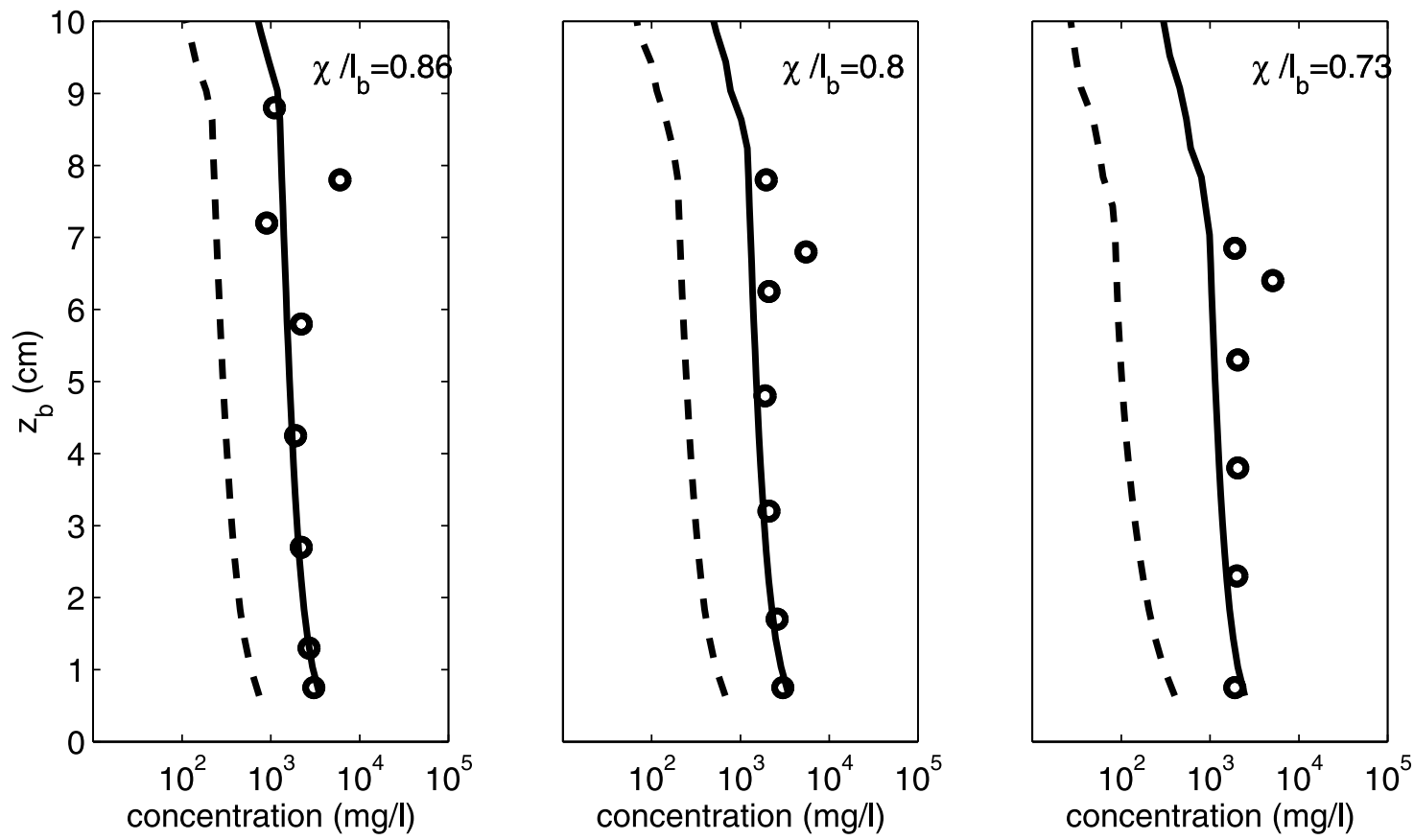

Figure 8. Comparisons of wave-averaged sediment concentration profiles using the single-phase model at three locations within the surf zone of Sato et al. [1990] with the same wave condition and sand particles in Figure 7. Open circles represent the measured data, dashed curves represent the results calculated with $e_{k}=0.0$, and solid curves represent that with $e_{k}=0.018$. 

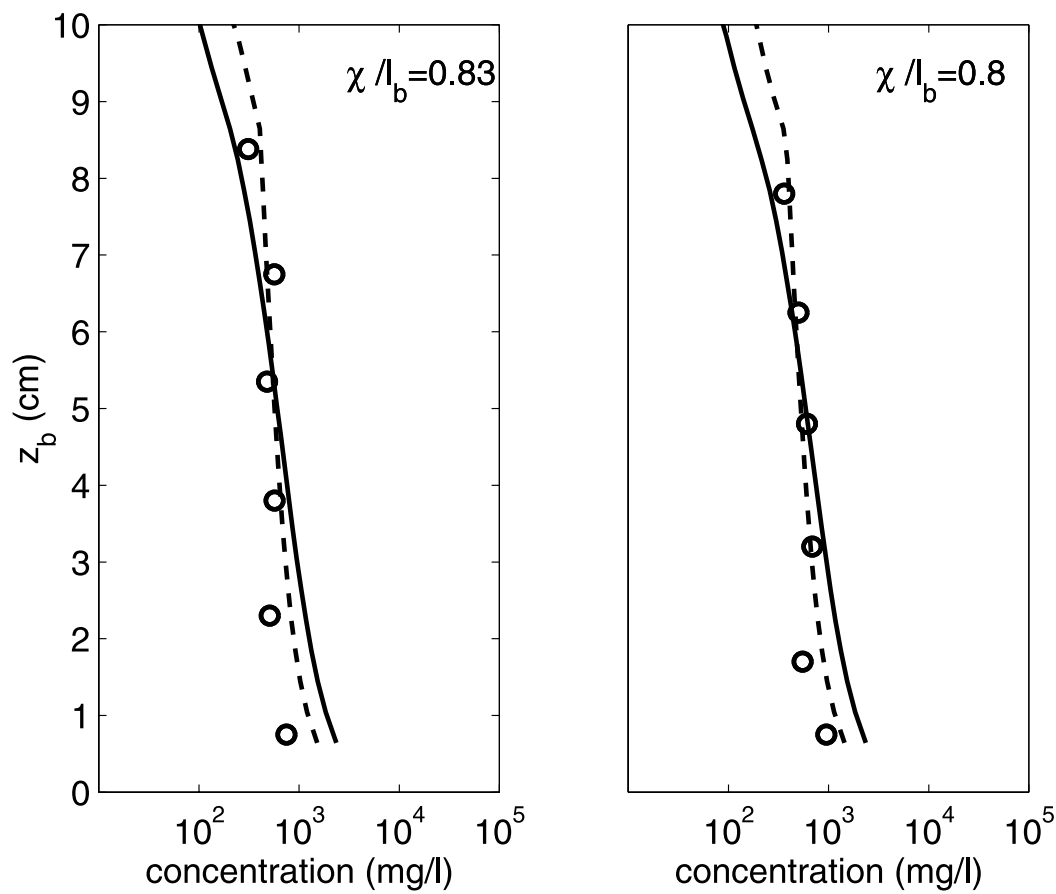

Figure 9. Comparisons of wave-averaged sediment concentration profiles at two locations within the surf zone of Sato et al. [1990] using the two-phase model (solid curves) and the single-phase model (dashed curves) with the same wave condition as in Figure 7 but using sand of diameter $d=0.18 \mathrm{~mm}$. Open circles represent the measured data.

depends on flow type and is greater than unity under surface gravity waves, which implies that the turbulent diffusion of sediment is less efficient than that of fluid momentum.

[45] On the basis of the measurements by Coleman [1981], van Rijn [1984b] suggests that the sediment diffusivity should always be greater than the eddy viscosity. It is argued that for a sediment particle with specific gravity greater than unity, the centrifugal force on the particle would be greater than that on a fluid parcel as they are both agitated by the turbulent eddies, and hence causes larger sediment diffusivity. Recently, Chang and Cowen [2002] measure the diffusivity of neutrally buoyant particles in round jet, using the nonintrusive PTV-LIF technique. Their results also indicate that the eddy diffusivity is greater than eddy viscosity even for neutrally buoyant particles. From the theoretical point of view, the "large-scale sediment stresses" [e.g., Hsu et al., 2003, 2004], or the so-called the "turbophoresis" [e.g., Young and Leeming, 1997], or the "drift velocity" [Czernuszenko, 1998] is usually neglected in most of the sediment transport models (also in the present models). These three terminologies describe the identical physical feature that represents additional advective drift of sediment due to the gradient of mean square particle velocity fluctuations. The additional sediment drift may also enhance the transport of sediments. Therefore, for an approximated model that does not incorporate this mechanism it is not surprising that a Schmidt number smaller than unity is required so that the "apparent" sediment diffusivity is increased to match the measured data. We consider that the argument for a Schmidt number that is smaller than unity is physically sound.

[46] As for the reason that under surface gravity waves the Schmidt number is required to be greater than unity in the single-phase approach, we believe that it is perhaps a consequence of oversimplifications in the single-phase description of sediment transport. It is clear that there are some fundamental differences between the governing equations of a two-phase approach and a single-phase approach. In the two-phase approach, various forces that are responsible for the movement of sediments are calculated in the sediment momentum equations and hence determine the sediment fluxes $\bar{c} \tilde{u}^{S}$ and $\bar{c} \tilde{w}^{s}$; whereas the sediment concentration adjusts to the variations in sediment fluxes through the mass balance equation. On the other hand, in the singlephase approach, because a momentum equation for sediment is not available, various forces that control the sediment motion are phrased directly in a mass balance through a mass fluxes, resulting in the advection-diffusion equation. More specifically, the theoretical basis for the advection-diffusion equation comes from the Rouse [1937] type formulation (e.g., equation (15)) that balances the upward and downward fluxes of sediment, which is a plausible description for sediment transport under steady, fully developed flow condition. In order to describe sediment transport in the nearshore where the flows are rarely steady or uniform, the Rouse formula is further extended heuristically to include terms associated with temporal variation, advection and the flow inhomogeneity and render the advection-diffusion equation.

\section{Conclusion}

[47] The extension of a depth- and phase-resolving crossshore hydrodynamic model COBRAS to describe suspended sediment transport under waves, based on dilute two-phase approach and single-phase approach, is reported. The pickup 
function of van Rijn [1984a] is adopted for the near-bed sediment boundary condition. In the U tube and nonbreaking wave conditions, fairly good agreements with the measured wave-averaged concentration are achieved in both the single-phase and two-phase approaches by calibrating the Schmidt number. Nevertheless, the detailed time histories of the predicted sediment concentration are less satisfactory because of the limitations on the available sediment near-bed boundary conditions. We demonstrated clearly that when the measured time history of sediment concentration is used as the near-bed sediment boundary condition, the predicted instantaneous sediment concentrations above the bed become fairly accurate. This implies that the models describe the physics in the dilute region reasonably well.

[48] Using the calibrated Schmidt number in the nonbreaking wave case, we further test the models with the measured data of suspended sediment under shoaling broken waves of Sato et al. [1990]. In both the two-phase and single-phase approach, we find that in addition to bottom friction, the effects of breaking wave turbulence on the nearbed sediment pick-up need to be considered in order to achieve reasonable agreements with the measured waveaveraged sediment concentration within the surf zone.

[49] Because the Schmidt number in the single-phase model must be adjusted according to flow type, the twophase model is considered to be more rigorous and robust. However, because of the uncertainties in the present near-bed sediment boundary conditions, the single-phase approach remains a valuable alternative as an efficient computational tool for practical purpose. To obtain better near-bed sediment boundary conditions, future developments should focus on the understanding the small-scale sediment transport processes in the concentrated region through detailed experiment and modeling. Recently, significant progress has been made in terms of mathematical modeling on the small-scale sheet flow processes under waves [e.g., Dong and Zhang, 1999, 2002; Drake and Calantoni, 2001; Malarkey et al., 2003; Hsu et al., 2004]. Since most of these mathematical models are based on solving the complete two-phase equations, any new findings and parameterizations on an improved boundary conditions developed from these small-scale models should be directly and consistently implemented in the present dilute twophase model.

\section{Notation}

$\bar{c}$ ensemble-averaged sediment (volume) concentration.

$c^{\prime}$ concentration fluctuation, $c^{\prime}=c-\bar{c}$.

$\tilde{u}_{i}^{f}$ Favre-averaged fluid velocity.

$\tilde{u}_{i}^{s} \quad$ Favre-averaged sediment velocity.

$\Delta u_{i}^{f}$ fluid velocity fluctuation, $\Delta u_{i}^{f}=u^{f}-\tilde{u}_{i}^{f}$.

$\tilde{u}^{f}$ Favre-averaged fluid horizontal velocity.

$\tilde{w}^{s} \quad$ Favre-averaged sediment vertical velocity.

$\bar{P}^{f}$ ensemble-averaged fluid pressure.

$\tau_{i j}^{f}$ fluid stresses, including viscous and Reynolds stresses.

$k_{f}$ fluid turbulence kinetic energy.

$\epsilon_{f}$ fluid turbulence dissipation rate.

$\nu_{f t}$ fluid turbulent eddy viscosity.

$g_{2}$ gravitational acceleration, $-9.8 \mathrm{~m}^{2} / \mathrm{s}$, with $g=\left|g_{2}\right|$.

$\rho^{f}$ fluid density. $\rho^{s} \quad$ sediment density.

$v_{f}$ fluid kinematic viscosity.

$\mu_{f}$ fluid dynamic viscosity.

$d$ particle diameter.

$s$ particle specific gravity.

$W_{0}$ particle fall velocity.

$\sigma_{c} \quad$ Schmidt number.

$K_{s}$ roughness.

$\kappa \quad$ Karman constant, 0.41 .

$e_{k}$ sediment suspension efficiency.

$u_{*}$ bed friction velocity.

$\theta$ Shields parameter.

$\phi_{p}$ sediment pickup function.

$T_{p}$ particle response time.

$T_{L}$ fluid turbulence timescale.

$H$ wave height.

$T$ wave period.

$h$ water depth.

$l_{b} \quad$ surf zone length.

[50] Acknowledgments. This research has been supported by NSF grants CTS-0000675 and OCE-0095834 to Cornell University. This paper is also a resulting product [R/CCP-9] funded under award NA16RG1645 from the National Sea Grant College Program of U.S. Department of Commerce's National Oceanic and Atmospheric Administration to the Research Foundation of State University of New York on behalf of New York Sea Grant. The financial supports for Tian-Jian Hsu provided by Department of Civil and Environmental Engineering, University of Delaware, and the Coastal Ocean Institute of Woods Hole Oceanographic Institution are also acknowledged. Woods Hole Oceanographic Institution contribution 11060 .

\section{References}

Chang, K.-A., and E. A. Cowen (2002), Turbulent Prandtl number in a neutrally buoyant turbulent round jet, J. Eng. Mech., 128, 1082-1087.

Coleman, N. L. (1981), Velocity profiles with suspended sediment, J. Hydraul. Res., 19, 211-229.

Czernuszenko, W. (1998), Drift velocity concept for sediment-laden flows, J. Hydraul. Eng., 124, 1026-1033.

Deigaard, R., J. Fredsøe, and I. B. Hedegaard (1986), Suspended sediment in the surf zone, J. Waterw. Port Coastal Ocean Eng., 112, 115-128.

Dohmen-Janssen, C. M., and D. M. Hanes (2002), Sheet flow dynamics under monochromatic nonbreaking waves, J. Geophys. Res., 107(C10), 3149, doi:10.1029/2001JC001045.

Dong, P., and K. Zhang (1999), Two-phase flow modeling of sediment motions in oscillatory sheet flow, Coastal Eng., 36, 87-109.

Dong, P., and K. Zhang (2002), Intense near-bed sediment motions in waves and currents, Coastal Eng., 45, 75-87.

Drake, T. G., and J. Calantoni (2001), Discrete particle model for sheet flow sediment transport in the nearshore, J. Geophys. Res., 106, 19,85919,868 .

Duy, N. T., and T. Shibayama (1997), A convection-diffusion model for suspended sediment in the surf zone, J. Geophys. Res., 102, 23,169$23,186$.

Elghobashi, S. E., and T. W. Abou-Arab (1983), A two-equation turbulence model for two-phase flows, Phys. Fluids, 26, 931-938.

Fredsøe, J., and R. Deigaard (1992), Mechanics of Coastal Sediment Transport, World Sci., River Edge, N. J.

Hagatun, K., and K. Eidsvik (1986), Oscillating turbulent boundary layer with suspended sediments, J. Geophys. Res., 91, 13,045-13,055.

Horikawa, K., A. Watanabe, and S. Katori (1982), Sediment transport under sheet flow condition, in Coastal Engineering 1982, edited by B. L. Edge, pp. 1335-1352, Am. Soc. of Civ. Eng., Reston, Va.

Hsu, T.-J. (2002), A two-phase flow approach for sediment transport, Ph.D. thesis, Cornell Univ., Ithaca, N. Y.

Hsu, T. J., J. T. Jenkins, and P. L.-F. Liu (2003), On two-phase sediment transport: Dilute flow, J. Geophys. Res., 108(C3), 3057, doi:10.1029/ 2001JC001276

Hsu, T.-J., J. T. Jenkins, and P. L.-F. Liu (2004), On two-phase sediment transport: Sheet flow of massive particles, Proc. R. Soc. London, Ser. A, 460(2048), doi:10.1098/rspa.2003.1273.

Jenkins, J. T., and D. M. Hanes (1998), Collisional sheet flows of sediment driven by turbulent fluid, J. Fluid Mech., 370, 29-52. 
Karambas, T. V., and C. Koutitas (2002), Surf and swash zone morphology evolution induced by nonlinear waves, J. Waterw. Port Coastal Ocean Eng., 128, 102-113.

Kobayashi, N., and B. D. Johnson (2001), Sand suspension, storage, advection and settling in surf and swash zones, J. Geophys. Res., 106, 9363-9376

Kobayashi, N., and Y. Tega (2002), Sand suspension and transport on equilibrium beach, J. Waterw. Port Coastal Ocean Eng., 128, 238-248.

Li, Z., and A. G. Davies (1996), Toward predicting sediment transport in combined wave-current flow, J. Waterw. Port Coastal Ocean Eng., 122, $157-164$.

Lin, P., and P. L.-F. Liu (1998a), A numerical study of breaking waves in the surf zone, J. Fluid Mech., 359, 239-264.

Lin, P., and P. L.-F. Liu (1998b), Turbulence transport, vorticity dynamics, and solute mixing under plunging breaking waves in surf zone, J. Geophys. Res., 103, 15,677-15,694.

Lin, P., and P. L.-F. Liu (2004), Discussion of "Vertical variation of the flow across the surf zone," Coastal Eng., 50, 161-164.

Lynett, P., T.-R. Wu, and P. L.-F. Liu (2002), Modeling wave runup with depth-integrated equations, Coastal Eng., 46, 89-107.

Malarkey, J., A. G. Davies, and Z. Li (2003), A simple model of unsteady sheet-flow sediment transport, Coastal Eng., 48, 171-188.

McLean, S. R. (1992), On the calculation of suspended load for noncohesive sediments, J. Geophys. Res., 97, 5759-5770.

Nielsen, P. (1992), Coastal Bottom Boundary Layers and Sediment Transport, World Sci., River Edge, N. J.

Pedersen, C., R. Deigaard, J. Fredsøe, and E. Hansen (1995), Simulation of sand in plunging breakers, J. Waterw. Port Coastal Ocean Eng., 121, $77-87$.

Rakha, K. A., R. Deigaard, and I. Broker (1997), A phase-resolving cross shore sediment transport model for beach profile evolution, Coastal Eng., $31,231-261$.

Ribberink, J. S., and A. A. Al-Salem (1995), Sheet flow and suspension of sand in oscillatory boundary layers, Coastal Eng., 25, 205-225.

Richardson, J. F., and W. N. Zaki (1954), Sedimentation and fluidization, part 1, Trans. Inst. Chem. Eng., 32, 35-53.

Rouse, H. (1937), Modern conceptions of the mechanics of turbulence, Trans. Am. Soc. Civ. Eng., 102, 463-543.
Sato, S., K. Homma, and T. Shibayama (1990), Laboratory study on sand suspension due to breaking waves, Coastal Eng. Jpn., 33, $219-231$

Savioli, J., and P. Justesen (1996), Sediment in oscillatory flows over a plane bed, J. Hydrogr. Res., 35, 177-190.

Squires, K. D., and J. K. Eaton (1994), Effect of selective modification of turbulence on two-equation models for particle-laden turbulent flows, J. Fluid Eng., 116, 778-784.

Staub, C., I. G. Jonsson, and I. A. Svendsen (1996), Sediment suspension in oscillatory flow: Measurement of instantaneous concentration at high shear, Coastal Eng., 27, 67-96.

Sumer, B. M., A. Kozakiewicz, J. Fredsøe, and R. Deigaard (1996), Velocity and concentration profiles in sheet-flow layer of movable bed, J. Hydrogr. Eng., 122, 549-558.

Ting, F. C. K., and J. T. Kirby (1995), Dynamics of surf-zone turbulence in a strong plunging breaker, Coastal Eng., 24, 177-204.

Ting, F. C. K., and J. T. Kirby (1996), Dynamics of surf-zone turbulence in a spilling breaker, Coastal Eng., 27, 131-160.

van Rijn, L. C. (1984a), Sediment pick-up function, J. Hydraul. Eng., 110, $1494-1502$

van Rijn, L. C. (1984b), Sediment transport, part II: Suspended load transport, J. Hydraul. Eng., 110, 1613-1641.

Wei, G., and J. T. Kirby (1995), A time-dependent numerical code for extended Boussinesq equations, J. Waterw. Port Coastal Ocean Eng., 121, 251-261.

Wilson, K. C. (1987), Analysis of bed-load motion in high shear stress, J. Hydrogr. Eng., 113, 97-103.

Young, J., and A. Leeming (1997), A theory of particle deposition in turbulent pipe flow, J. Fluid Mech., 340, 129-159.

T.-J. Hsu, Applied Ocean Physics and Engineering Department, Woods Hole Oceanographic Institution, Woods Hole, MA 02543, USA. (thsu@ whoi.edu)

P. L.-F. Liu, School of Civil and Environmental Engineering, Cornell University, Ithaca, NY 14853, USA. (p113@cornell.edu) 